\title{
Outcomes of Ventriculo-peritoneal Shunt Based on Clinical Observation: Retrospective Study in Rural Area Developing Country Indonesia
}

\section{Agung Budi Sutiono*}

Department of Neurosurgery, Universitas Padjadjaran Hasan Sadikin Hospital, Indonesia

*Corresponding Author: Agung Budi Sutiono, Department of Neurosurgery, Universitas Padjadjaran Hasan Sadikin Hospital, Indonesia.
Received: November 16, 2021

Published: November 25, 2021

(C) All rights are reserved by Agung Budi

Sutiono.

\begin{abstract}
Introduction: Hydrocephalus is a condition where an abnormal build-up of cerebrospinal fluid (CSF) fluid causes an increase in pressure in the ventricles or subarachnoid space of the brain and one of the most common clinical conditions affecting the central nervous system. Although VP-shunt is a simple surgery, however the follow up post-operatively in rural city area especially in developing country like Indonesia has not been explore explicitly.

Methods: Retrospective study was conducted of 29 patient with ages ranged from 2 month until 25 years at Hasan Sadikin General Hospital rural city Bandung Indonesia.

Results: The result showed most of incidence is in males. The common type of hydrocephalus is communicant hydrocephalus with $61 \%$ cases. We also described correlation between ages and length of stay in hospital and the result is the youngest VP-shunt for hydrocephalus, the longer should stay in hospital $(\mathrm{p}<0,00)$. There was statistically significant difference in any outcome between adult patient and children $(\mathrm{p}<0,00)$ but there is no correlation between type of re-therapy (repair or removal shunt) with lenght of previous VP-shunt surgery $(\mathrm{p}=0,99)$.

Conclusion: We found that the younger patient need to stay longer in hospital than in adult patient. And the adult patient of hydrocephlus has better outcome than in children. Of course the outcome depends on multiple factors especially in children which are related to myelomeningocele or other congenital anomalies, therefore it need more investigation.
\end{abstract}

Keywords: Hydrocephalus; Ventriculoperitoneal Shunt; Rural; Outcomes

\section{Introduction}

Hydrocephalus is a condition where an abnormal build-up of cerebrospinal fluid (CSF) fluid causes an increase in pressure in the ventricles or subarachnoid space of the brain and one of the most common clinical conditions affecting the central nervous system. Prevalention has been estimated that it affects 0.9 to 1.5 per 1000 births and some of congenital abnormalities are included. It can be caused by either the blockage of CSF flow (obstructive/ non-communicating hydrocephalus) in the ventricular system or by inadequate reabsorption of CSF fluid (non-obstructive/communicating hydrocephalus). These features result in enlargement of the ventricles or subarachnoid space and increase intracranial pressure (ICP). The severity of ICP can compress surrounding brain parenchyma, manifesting into identifiable acute or chronic symptoms depending on the age of onset. Hydrocephalus has incidence of three to four per 1000 births for congenital hydrocephalus [1,2]. However, with the advent of shunt surgeries, these children can be assured of a near normal neurological development. A VP-shunt is a long, plastic tube that allows fluid to drain from the brain to another part of the body. This drainage prevents the increase in pressure on the brain caused by hydrocephalus. A VP shunt has at least 3 parts. The first part is the ventricular catheter, which goes into the brain. The second part is the valve. It controls the pressure within the brain. The third part is the distal catheter. It is un- 
derneath the skin and connects the other parts of the VP shunt to a space within the body, usually the abdominal cavity (also called the peritoneal cavity). Several complication and outcomes are possibly occur in the post VP-shunt patient, during long term follow up. Although VP-shunt is a simple surgery, however the follow up post-operatively in rural city area especially in developing country like Indonesia has not been explore explicitly.

\section{Methods and Results}

This retrospective study was conducted in Hasan Sadikin Hospital rural area Bandung Indonesia. The cases were evaluated for gender, type of hydrocephalus, correlation between ages and longstay, therapy and outcome. The patients were 29 patient ( 8 females and 21 males) with clinical diagnosis of hydrocephalus. The ages ranged from 2 month until 25 years. The mean age was 6,72 years. The result are in figure 1 showed demographic of hydrocephalus between males and female. Most of incidence of hydrocephalus is in males. The likely type of hydrocephalus in 29 cases like shown in figure 2 is communicant hydrocephalus. Hydrocephalus in young and middle aged adults is a unique and often confusing condition, the result for correlation between age and lenght of stay in the hospital after VP shunt is the youngest age who had hydrocephalus, then the longer lenght of stay in hospital after VP Shunt $(p<0,00)$ as shown in figure 3. VP-shunt replacement due to infection were reported in previous research, this is probably explain that impossible to remove VP Shunt in patient with vp shunt for longer time than a short time (Figure 4). Outcome in shunt for hydrocephalus included clinical outcome and surgical outcome. Statistically adult has better outcome than children. But there is no correlation between type of therapy with outcomes $(p=0,99)$ (Figure 5).

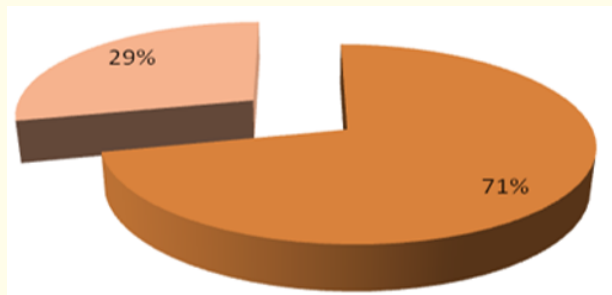

$$
\begin{aligned}
& =\text { Male } \\
& =\text { Female }
\end{aligned}
$$

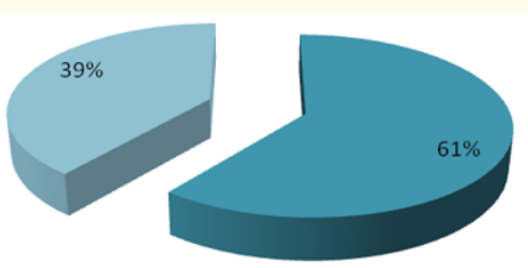

ㅁ.

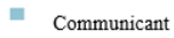
Non communicant

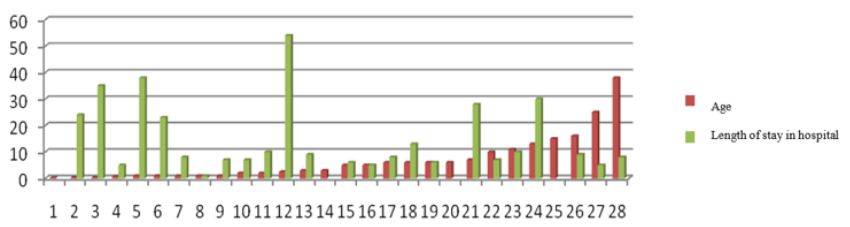

Figure 3: Correlation diagram based on age and length of

$$
\text { stay }(\mathrm{p}<0,00) \text {. }
$$

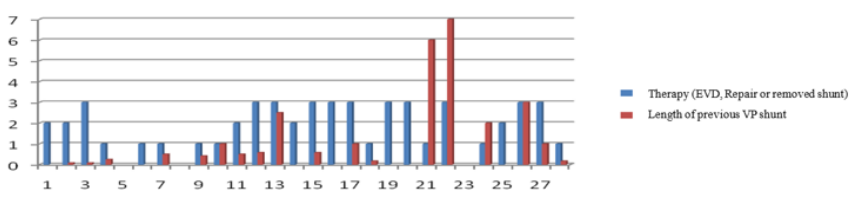

Figure 4: Correlation diagram of therapy and length of previous VP-shunt $(\mathrm{P}<0.99)$.

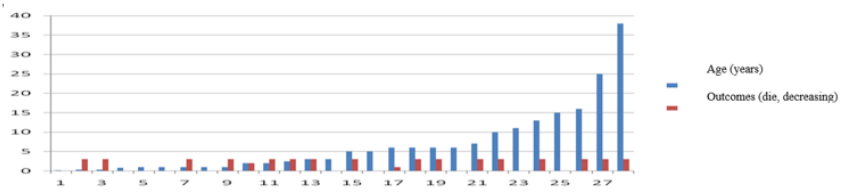

Figure 5: Correlation diagram based on age and outcomes $(\mathrm{P}<0.00)$.

Figure 1: Diagram distribution of gender. 


\section{Discussion}

The true incidence of hydrocephalus in children and adults is unknown. It has been estimated that it affects 0.9 to 1.5 per 1000 births. When congenital abnormalities are included (e.g. spina bifida, myelomeningocele), hydrocephalus can affect 1.3 to 2.9 per 1000 births. ${ }^{1}$ In our patient series, the most incidence in hydrocephalus is males but, from the past literature, Generally, incidence is equal in males and females [3]. There are two type of hydrocephalus. First is called non-communicating or obstructive hydrocephalus which is obstruction of the CSF pathway if the ventricles no longer provide free passage of CSF through them into the subarachnoid space. Another type of hydrocephalus is communicating or extraventricular obstructive hydrocephalus. It usually results from a thickening of the arachnoid around the base of the brain, which blocks the flow of CSF from the spinal to the cortical subarachnoid spaces. There is no researched found comparation result between non-communicating hydrocephalus and communicating hydrocephalus but, our result showed that communicant hydrocephalus showed the most cases overall. Although hydrocephalus is almost always treated successfully with surgical placement of a shunt, shunt malfunction and, less frequently, infection occur in many cases. Shunt malfunction, which is caused by obstruction, simply means that the shunt is not able to divert enough CSF away from the ventricles in the brain. These are serious problems and must be treated appropriately. Another problem is Infections. People with VP shunts are at risk of developing a shunt infection secondary to abdominal infection. In either case, the shunt infection must be treated immediately to avoid life-threatening illness or possible brain damage [4]. Farid., et al. reported that median time to shunt malfunction was severely affected by extreme of age. Most of the shunt failures occurred within 6 months post shunt placement, which is compatible with previous reports from developed countries. The VP shunt failure rate reported earlier ranged from $18 \%$ to $29 \%$ for adult hydrocephalus [5]. The overall VP shunt failure rate $(15.4 \%)$ that report is consistent with the shunt failure rate $(15.2 \%)$ recently recounted by Reddy., et al. The shunt failure rates at 6 months, i.e. $19 / 227$ (8.37\%), and at 500 days, i.e. 26/227 (11.45\%), are well below the previously reported failure rates. In another study, Reddy., et al. reported 32\% incidence of shunt revision in adult hydrocephalus patients [6]. This might be accounted for by the fact that elderly patients have fragile and atrophic brain parenchyma. Surgical intervention in such patients was probably associated with a higher risk of iatrogenic trauma inflicted to the nearby tissues while placing the VP shunt. Injury to cells of the choroid plexus within the ventricles could lead to the accumulation of cellular debris within the catheter and block the tubing of the VP shunt, resulting in shunt blockage [7]. Although this explanation seems plausible theoretically, it cannot be said with certainty that this was the actual reason for early shunt failure in elderly patients. Moreover, the result from farid., et al. Patients with increased age, prolonged hospital stay. This is quite different with our result which is children should stay longer in hospital after malfunction of VP shunt. Furthermore, ibrahim., et al. also reported that infection rate were higer in replacement vp shunt [8].

\section{Conclusion}

In conclusion, we found that the younger patient need to stay longer in the hospital than in adult patient. And the adult patient of hydrocephlus has better outacome than in children. Of course the outcome depends on multiple factors especially in children which are related to myelomeningocele or other congenital anomalies, therefore it need more investigation.

\section{Bibliography}

1. Beni-Adani L., et al. "The occurrence of obstructive vs. absorptive hydrocephalus in newborns and infants: relevance to treatment choices". Child's Nervous System 22.12 (2006): 1542-1563.

2. Ahmed A., et al. "Outcome analysis of shunt surgery in hydrocephalus". Journal of Indian Association of Pediatric Surgeons 14.3 (2009).

3. Gautam VK., et al. "Reviewing Hydrocephalus: Personal experience of a Neurosurgeon with Ventriculoperitoneal shunt treatment". International Journal of Medicine 2.2 (2014): 56-59.

4. "Hydrocephalus diagnosed in young and middle-age Adult". Hydrocephalus association. San francisco. (2013).

5. Lam CH and Villemure JG. "Comparison between ventriculoatrial and ventriculoperitoneal shunting in the adult population". British Journal of Neurosurgery 11 (1997): 43-48.

6. Reddy GK., et al. "Ventriculoperitoneal shunt surgery outcome in adult transition patients with pediatric-onset hydrocephalus". Neurosurgery 70 (2012): 380-389.

7. Farid K., et al. "Factor affecting ventriculoperitoneal shunt survival in adult patients". Surgical Neurology International 6.25 (2015). 
8. Ibrahim SO., et al. "When should Ventrikuloperitoneal Shunt Placement be Performed in Cases with Myelomeningocele and Hydrocephalus?". Turkish Neurosurgery 18.4 (2008): 387-391.

Volume 4 Issue 12 December 2021

(C) All rights are reserved by Agung Budi Sutiono. 